\title{
Bandwidth-Efficient Multicast in Heterogeneous Wireless Networks
}

\author{
De-Nian Yang and Ming-Syan Chen \\ Department of Electrical Engineering \\ National Taiwan University \\ Taipei, Taiwan, R.O.C. \\ Email: tony@kiki.ee.ntu.edu.tw; mschen@cc.ee.ntu.edu.tw
}

\begin{abstract}
In this paper, we propose a bandwidth-efficient multicast mechanism for heterogeneous wireless networks. We reduce the bandwidth cost of a shortest-path IP multicast tree by adaptively selecting the cell and the wireless technology for each mobile host to join the multicast group. Our mechanism enables more mobile hosts to cluster together and lead to the use of fewer cells to save the scarce wireless bandwidth. Besides, the paths in the multicast tree connecting to the selected cells share more common links to save the wireline bandwidth. We formulate the selection of the cell and the wireless technology for each mobile host in the heterogeneous wireless networks as an optimization problem. We use Integer Linear Programming to model the problem and design an distributed algorithm based on Lagrangean relaxation. Our mechanism supports the dynamic group membership and offers mobility of group members. Moreover, our mechanism requires no modification on the current IP multicast routing protocols.
\end{abstract}

\section{INTRODUCTION}

The success of wireless and mobile communications in the 21 st century has resulted in a large variety of wireless technologies, such as second- and third-generation cellulars, satellite, WiFi, and Bluetooth. The heterogeneous wireless networks combine various wireless networks and provide universal wireless access. Users in the heterogeneous wireless networks are usually covered by more than one cell to avoid connection drop and service disruption. Besides, more mobile terminals in the wireless networks are likely to own multiple wireless technologies. Therefore, the heterogeneous wireless networks provide the mobile hosts with many choices for the cell and the wireless technologies to access the Internet.

Multicast is an efficient way for one-to-many and manyto-many communications. Each multicast group owns a set of members, and each member can be a sender or a receiver of the group. The sender in a multicast group delivers data in a multicast tree to all receivers of the group. Current IP multicast routing protocols adopt the shortestpath trees for data delivery [1][2][4][3][5]. The path from the root of a shortest-path tree to each member must be the shortest path in the network. In other words, the routing of a shortest-path tree is fixed once the root and all group members have been determined. As a consequence, the bandwidth consumption in an IP multicast tree will not be able to be reduced in wired networks.

In this paper, we first comment that the bandwidth consumption in a shortest-path tree can be reduced in the

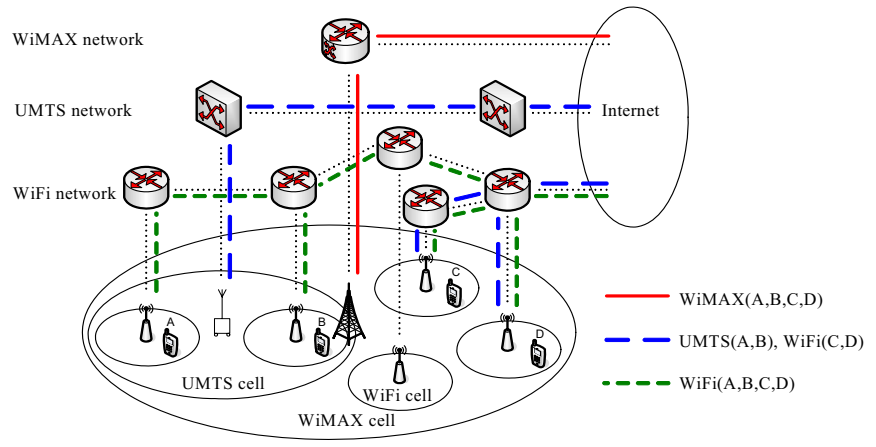

Fig. 1. An example that provides three different multicast trees by selecting different cells and wireless technologies for mobile hosts.

heterogeneous wireless networks because the routing of a shortest-path tree here is more flexible. A shortest-path tree in the heterogeneous wireless networks consists of two parts. The first one is composed of the cell and the wireless technology chosen by each mobile host. The second one comprises the wired links that connect the root of the tree and the chosen cells. Therefore, we can change the routing of a shortest-path tree by selecting different cells and wireless technologies for the mobile hosts to reduce the bandwidth consumption. Consider the scenario in Fig. 1 as an example, where mobile hosts A, B, C, and D are the members of the multicast group. The example presents three different shortest-path trees to serve the four mobile hosts. The first one uses a WiMAX cell to serve the four mobile hosts. The second one uses a UMTS cell to serves mobile hosts $\mathrm{A}$ and $\mathrm{B}$ and two $\mathrm{WiFi}$ cells to serve mobile hosts $\mathrm{C}$ and $\mathrm{D}$. The third one uses four WiFi cells to serves the four mobile hosts. Therefore, this example shows that the routing of a shortest-path tree in the heterogeneous wireless networks is not unique.

As we know, there is no related work about the selection of the cell and the wireless technology for each mobile host to build a bandwidth-efficient multicast tree in the heterogeneous wireless networks. Previous works for mobile multicast in the heterogeneous wireless networks focus on the efficient mechanisms to provide seamless handover between different networks [6][7][8][9] and the related security issues [10]. Previous works have proposed efficient mechanisms for the $3 \mathrm{G}$ wireless networks to provide multi- 
cast services [11][12][13][14][15], irrelevant to the routing of a shortest-path tree. Consequently, in this paper, we propose a mechanism to reduce the bandwidth consumption in a shortest-path tree by adaptively selecting the cell and the wireless technology for each mobile host in the heterogeneous wireless networks. The feature distinguishes our work from others.

Explicitly, we formulate in this paper the selection of the cell and the wireless technology for each mobile host as an optimization problem, which is denoted as the Cell and Technology Selection Problem (CTSP) in the heterogeneous wireless networks for multicast communications. The problem is to select the cell and the wireless technology for each group member to minimize the total bandwidth cost of the shortest-path tree. We design a mechanism, which includes an Integer Linear Programming (ILP) formulation and a distributed algorithm, to solve the CTSP. The network operator can use our ILP formulation to find the optimal solution for network planning. Our algorithm LAGRANGE, which is based on Lagrangean relaxation [19] on our ILP formulation. Our algorithm decomposes the original problem into multiple sub-problems such that each sub-problem can be solved by each member and base station individually. In other words, our algorithm can be implemented in the distributed manner. In addition, our algorithm can be regarded as a rerouting algorithm. Therefore, our algorithm requires sophisticated handover protocols such as the related works [16][17][18].

Our mechanism has the following advantages. First, for each wireless technology, our mechanism reduces the number of cells used in a shortest-path tree. Our mechanism clusters the mobile hosts such that nearby mobile hosts tend to use the same cell. Second, our mechanism considers various aspects to select the cell and the wireless technology for each mobile host. For a set of nearby mobile hosts, our mechanism uses a single larger cell or multiple smaller cells to serve these mobile hosts, depending on the number of mobile hosts, the location of each mobile host, and the bandwidth cost of each wireless technology. Third, our mechanism is transparent to the IP multicast routing protocols. The shortest-path tree is created by each member joining the multicast group with the IP multicast routing protocols, after each member selects the cell and the wireless technology according to our mechanism. We thereby require no modification on the current IP multicast routing protocols.

The rest of the paper is as follows. Section II describes our assumption and presents our ILP formulation. We propose algorithm LAGRANGE based on Lagrangean relaxation in Section III. Finally, we conclude our paper in Section IV.

\section{Problem Description}

In this paper, we consider the Cell and Technology Selection Problem (CTSP) in the heterogeneous wireless networks for multicast communications. The problem is to select the cell and the wireless technology for each group member to minimize the total bandwidth cost of the shortest-path tree. The total bandwidth cost of a shortestpath tree consists of the total wireless bandwidth cost of the selected cells and the total wireline bandwidth cost of the shortest-path tree spanning the root and each selected cells.

The mobile hosts considered in this paper are the members of a multicast group. A cell covers a mobile host if the mobile host is within the transmission range of the base station of the cell. Let a cell be a candidate cell if the cell covers at least one mobile host. A node or link $x$ is downstream to another node or link $y$ in the shortest-path tree if $y$ is on the path from the root of the tree to $x$. A sub-tree that is downstream to a link $e$ contains link $e$ and every node and link that are downstream to $e$ in the shortestpath tree. For simplicity, the selection of the cell for each mobile host means the selection of both the cell and the wireless technology in the rest of the paper.

The notation in this paper is summarized as follows,

$C$ set of cells in the heterogeneous wireless networks;

$E \quad$ set of links in the shortest path from each candidate cell to the root of the tree;

$M \quad$ set of mobile hosts in the network;

$M_{c} \quad$ set of mobile hosts covered by cell $c, c \in C$;

$C_{m} \quad$ set of cells covering mobile host $m, m \in M$, $C_{m} \subseteq C$; if mobile host $m$ selects cell $c$ manually, we let the set $C_{m}$ contain only a cell $c$;

$C_{u} \quad$ set of cells that are downstream to node $u$ in the shortest-path tree, $C_{u} \subseteq C$;

$E_{c} \quad$ set of links in the shortest path from cell $c$ to the root of the tree, $E_{c} \subseteq E$;

$E_{u} \quad$ set of links that are downstream to node $u$ in the shortest-path tree, $E_{u} \subseteq E$;

$e_{u, v} \quad$ the link from node $u$ to $v, e_{u, v} \in E$;

$b_{c} \quad$ bandwidth cost of cell $c, c \in C$;

$b_{u, v} \quad$ bandwidth cost of link $e_{u, v}, e_{u, v} \in E$;

$c_{u, v}$ the cell with the base station $v$ connected to link $e_{u, v}, c_{u, v} \in C, e_{u, v} \in E$;

$r \quad$ the root of the shortest-path tree.

We use ILP to model the CTSP. The ILP formulation can find the optimal shortest-path tree in the heterogeneous wireless networks with any existing commercial software. Our ILP formulation has the following variables,
$\pi_{m, c} \quad$ a binary variable; $\pi_{m, c}$ is one if mobile host $m$ selects cell $c, m \in M, c \in C_{m}$;
$\sigma_{c} \quad$ a binary variable; $\sigma_{c}$ is one if cell $c$ is used in the shortest-path tree, $c \in C$;
$\lambda_{u, v} \quad$ a binary variable; $\lambda_{u, v}$ is one if link $e_{u, v}$ is used in the shortest-path tree, $e_{u, v} \in E$.

The objective function of our integer linear programming formulation is as follows,

$$
\min \sum_{c \in C} b_{c} \times \sigma_{c}+\sum_{e_{u, v} \in E} b_{u, v} \times \lambda_{u, v} .
$$

The constraints of our ILP formulation are as follows, 


$$
\begin{gathered}
\sum_{c \in C_{m}} \pi_{m, c}=1, \forall m \in M, \\
\pi_{m, c} \leq \sigma_{c}, \forall m \in M, \forall c \in C_{m}, \\
\sigma_{c} \leq \lambda_{u, v}, \forall c \in C, \forall e_{u, v} \in E_{c} .
\end{gathered}
$$

The first constraint guarantees that each mobile host selects one cell. The second constraint enforces that a cell is used in the shortest-path tree if it is selected by any mobile host. The third constraint states that a link is used in the shortest-path tree if it is on the path from any selected cell to the root of the tree. In addition to the above constraints, the problem has the constraints enforcing that $\pi_{m, c}, \sigma_{c}$, and $\lambda_{u, v}$ are all binary variables. We regard a set of selected cells that obey the above constraints as a feasible solution to the CTSP.

\section{Design of Algorithm LAGRANGE}

In this section, we propose an algorithm LAGRANGE. The algorithm is based on Lagrangean relaxation on our ILP formulation proposed in Section II. The algorithm relaxes a constraint of our ILP formulation and transfers the CTSP into the Lagrangean Relaxation Problem (LRP). The LRP owns a new objective function with the Lagrange multipliers and fewer constraints such that we can decompose the LRP into multiple sub-problems, where each subproblem can be solved in the distributed manner. The members in our algorithm collaboratively construct the shortest-path tree according to the solutions to the subproblems. Besides, the cost of each cell for each member is updated iteratively to reduce the total bandwidth cost of a shortest-path tree according to the current group membership and the locations of members.

The algorithm relaxes the second constraint in the ILP formulation to transfer the CTSP into the LRP, and the objective function of the LRP is as follows,

$$
\begin{gathered}
\min \sum_{c \in C} b_{c} \times \sigma_{c}+\sum_{e_{u, v} \in E} b_{u, v} \times \lambda_{u, v}+ \\
\sum_{m \in M} \sum_{c \in C_{m}} \mu_{m, c}\left(\pi_{m, c}-\sigma_{c}\right) \\
=\min \left[\sum_{m \in M} \sum_{c \in C_{m}} \mu_{m, c} \pi_{m, c}\right]+ \\
{\left[\sum_{c \in C}\left(b_{c}-\sum_{m: c \in C_{m}} \mu_{m, c}\right) \sigma_{c}+\sum_{e_{u, v} \in E} b_{u, v} \times \lambda_{u, v}\right],}
\end{gathered}
$$

where $\mu_{m, c}$ is the Lagrange multiplier, $\mu_{m, c} \geq 0, \forall m \in M$, $\forall c \in C_{m}$. The Lagrange multiplier is $\mu_{m, c}$ is the cost of cell $c$ for mobile host $c$. The LRP includes the first and the third constraints. The optimal solution to the LRP provides a lower bound on the objective value of the optimal solution to the CTSP, which is the total bandwidth cost of the optimal shortest-path tree.

We solve the LRP by decomposing the LRP into two sub-problems. We divide the objective function and the constraints of the LRP into two parts, where each subproblem owns one part of the objective function and constraints. The variables in the two sub-problems are mutually independent such that we can solve each sub-problem individually, and the solution to the LRP is just the combination of the solutions to the two sub-problems.
The objective function of the first sub-problem is as follows,

$$
\min \sum_{m \in M} \sum_{c \in C_{m}} \mu_{m, c} \pi_{m, c} .
$$

The first sub-problem has the following constraint,

$$
\sum_{c \in C_{m}} \pi_{m, c}=1, \forall m \in M
$$

In the sub-problem, each cell $c$ is associated with a cost $\mu_{m, c}$ for each mobile host $m$. The optimal solution to the first sub-problem is to find the cell with the minimum cost for each mobile $m$. The running time of the algorithm for the first sub-problem is thereby $O(|M||C|)$. In algorithm LAGRANGE, the cost $\mu_{m, c}$ for cell $c$ is stored in each mobile host $m$, and each mobile host thereby can find the cost with the minimum cost individually.

The objective function of the second sub-problem is as follows,

$$
\min \sum_{c \in C}\left(b_{c}-\sum_{m: c \in C_{m}} \mu_{m, c}\right) \sigma_{c}+\sum_{e_{u, v} \in E} b_{u, v} \times \lambda_{u, v} .
$$

The second sub-problem has the following constraint,

$$
\sigma_{c} \leq \lambda_{u, v}, \forall c \in C, \forall e_{u, v} \in E_{c} .
$$

In the sub-problem, each cell $c$ is associated with a profit $\sum_{m: c \in C_{m}} \mu_{m, c}$. If we choose a cell $c$, we acquire the profit but pay the wireless bandwidth cost $b_{c}$ and wireline bandwidth cost $b_{u, v}$ of each link $e_{u, v}$ on the shortest path from $c$ to the root. However, the wireline bandwidth $\operatorname{cost} b_{u, v}$ for each link $e_{u, v}$ can be shared by all downstream selected cells. Therefore, the objective function of the second problem is to minimize the net cost of all selected cells in the shortestpath tree that spans all candidate cells, and we have to find the best trade-off to select the cells.

Let $\delta_{u, v}$ denote the minimum net cost of the sub-tree that includes link $e_{u, v}$ and the sub-tree rooted at $v$. To find the minimum net cost of the whole shortest-path tree, we consider each link of the shortest-path tree in the bottomup manner. For the link $e_{u, v}$ that connects to cell $c_{u, v}$, the minimum net cost $\delta_{u, v}$ is as follows,

$$
\delta_{u, v}=\min \left\{0, b_{c_{u, v}}+b_{u, v}-\sum_{m: c_{u, v} \in C_{m}} \mu_{m, c_{u, v}}\right\},
$$

where the zero net cost corresponds to the case that cell $c_{u, v}$ is not selected, and net cost $b_{c_{u, v}}+b_{u, v}-\sum_{m: c_{u, v} \in C_{m}} \mu_{m, c_{u, v}}$ corresponds to the case that cell $c_{u, v}$ is selected. Therefore, net cost $\delta_{u, v}$ is guaranteed to be non-positive. Afterward, for each of the upstream links $e_{u, v}$, we derive the minimum net $\operatorname{cost} \delta_{u, v}$ from the minimum net cost of each downstream link $\delta_{v, w}$,

$$
\delta_{u, v}=\min \left\{0, b_{u, v}+\sum_{w: e_{v, w} \in E} \delta_{v, w}\right\} .
$$

If $\delta_{u, v}$ is negative, note that the above equation allows only some downstream links of $e_{u, v}$ own the selected cells in 


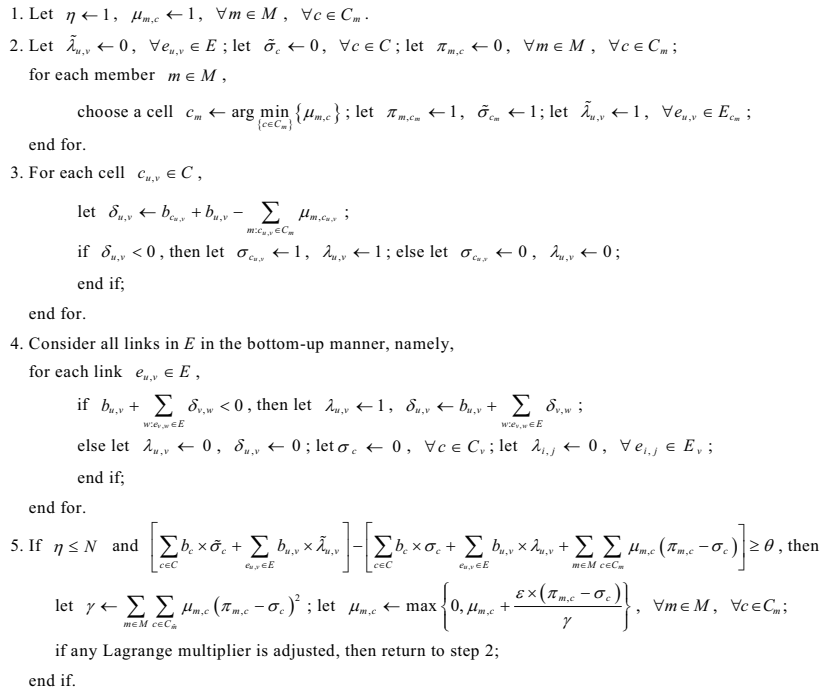

Fig. 2. Algorithm LAGRANGE.

the corresponding sub-trees, while the cells located in the sub-trees corresponding to other downstream links are not selected. We can find the minimum net cost of the shortestpath tree in $O(|E|)$ time.

Fig. 2 shows the details of our algorithm, where $\widetilde{\sigma}_{c}$ denote if algorithm LAGRANGE selects cell $c$, and $\widetilde{\lambda}_{u, v}$ denote if algorithm LAGRANGE uses link $e_{u, v}$ in the shortest-path tree. Initially, algorithm Lagrange assigns unit cost to each cell for each member in step one, and each member thereby can select any cell. Afterward, our algorithm iteratively reduces the total bandwidth cost of the shortest-path tree. At each iteration, our algorithm first finds the solution to the first sub-problem in step two. The algorithm then finds the solution to the second subproblem in step three and four. The algorithm then adjusts the cost of each cell for each member in step five such that we can find a shortest-path tree with lower bandwidth cost in the next iteration. Algorithm LAGRANGE stops when the number of iterations $\eta$ is larger than a threshold $N$, when our algorithm can no longer adjust the cost, or when the difference of the total bandwidth cost of the obtained shortest-path tree and the lower bound on the total bandwidth cost of the optimal shortest-path tree is within a threshold $\theta$. The parameter $\varepsilon$ in step five is a parameter that dominates the modification of the cost at each iteration in the subgradient algorithm. With a larger $\varepsilon$, the shortest-path tree improves faster, but the obtained shortest-path tree tends to consume more bandwidth than the obtained shortest-path tree with smaller $\varepsilon$. In this paper, we thereby reduce $\varepsilon$ as the improvement of the shortest-path tree becomes smaller.

\section{Conclusion}

In this paper, we propose a new mechanism to reduce the total bandwidth cost of a IP multicast tree by adaptively selecting the cell and the wireless technology for each mo- bile host. We model the selection of the cell and the wireless technology for each mobile host as an optimization problem. We use Integer Linear Programming (ILP) to formulate the optimization problem. The network operators can use our formulation for network planning. We also design an algorithm based on the Lagrangean relaxation. Our algorithm can be implemented as a network protocol that supports the dynamic group membership and the mobility of users.

\section{REFERENCES}

[1] D. Waitzman, C. Partridge, and S. Deering, "Distance vector multicast routing protocol," IETF RFC 1075, 1988.

[2] J. Moy, "Multicast extensions to OSPF," IETF RFC 1584, 1994.

[3] D. Estrin et al., "Protocol independent multicast-sparse mode (PIM-SM): protocol specification," IETF RFC 2117, 1997.

[4] A. Ballardie, "Core based trees (CBT version 2) multicast routing protocol specification," IETF RFC 2189, 1997.

[5] S. Bhattacharyya, "An overview of source-specific multicast (SSM)," IETF RFC 3569, 2003.

[6] T. G. Harrison C. L. Williamson, W. L. Mackrell, and R. B. Bunt, "Mobile multicast (MoM) protocol: multicast support for mobile hosts," ACM MOBICOM, 1997, pp. 151-160.

[7] C. R. Lin and K. -M. Wang, "Mobile multicast support in IP networks," IEEE INFOCOM, 2000, vol. 3, pp. 1664-1672.

[8] Y. Wang and W. Chen, "Supporting IP multicast for mobile hosts," ACM Mobile Networks and Applications," Jan. 2001, vol. 6 , no. 1 , pp. 57-66.

[9] J. -R. Lai, W. Liao, M. -Y. Jiang and C. -A. Ke, "Mobile multicast with routing optimization for recipient mobility," IEEE ICC 2001, vol. 5, pp. 1340-1344.

[10] Y. Sun, W. Trappe, and K. J. R. Liu, "A scalable multicast key management scheme for heterogeneous wireless networks," IEEE/ACM Transactions on Networking, Aug. 2004, vol. 12, no. 4, pp. 653-666.

[11] M. Hauge and O. Kure, "Multicast in 3G networks: employment of existing IP multicast protocols in UMTS," ACM International Workshop on Wireless Mobile Multimedia, 2002.

[12] R. Rummler and H. Aghvami, "End-to-end IP multicast for software upgrades of reconfigurable user terminals within IMT2000/UMTS networks," IEEE ICC, 2002, vol. 1, pp. 502-506.

[13] S. K. Palat, I. N. Weerasekera, and A. Casati, "Multicasting in UMTS," IEEE International Conference on 3G Mobile Communication Technologies, 2002, pp 96-101.

[14] U. Mudugamuwa, M. Karaliopoulos, R. Tafazolli, and B. Evans, "Reliable multicast transport and power scheduling for MBMS delivery over 3G mobile satellite systems," IEEE VTC, 2004, pp. 2836-2841.

[15] R. Rummler, Y. W. Chung, and A. H. Aghvami, "Modeling and Analysis of an Efficient Multicast Mechanism for UMTS," IEEE Transactions on Vehicular Technology, Jan. 2005, vol. 65, no. 1, pp. 350-365.

[16] K. Pahlavan et al., "Handoff in hybrid mobile data networks," IEEE Personal Communications, Apr. 2000, vol. 7, no. 2, pp. 34-47.

[17] J. McNair, I. F. Akyildiz and M. Bender "An intersystem handoff technique for IMT-2000 systems," IEEE INFOCOM, 2000, vol. 1, pp. 208-16.

[18] J. McNair and F. Zhu, "Vertical handoffs in fourth-generation multinetwork environments," IEEE Wireless Communications, Jun. 2004, vol. 11, no. 3, pp. 8-15.

[19] G. L. Nemhauser and L. A. Wosley, "Integer and combinatorial optimization," Wiley-Interscience series in discrete mathematics and optimization, 1999. 